\title{
EX-PRESS shunt implantation in Chinese glaucoma patients: 1-year results
}

Jennifer WH Shum ${ }^{1}$, FCOphthHK (Ophth), Bonnie NK Choy ${ }^{l}$, FRCSEd (Ophth), Jonathan CH Chan ${ }^{l}$, FRCSEd (Ophth), Wing Lau Ho ${ }^{2}$, FRCSEd (Ophth), Stephanie Cheri Woo ${ }^{3}$, MBBS, Jimmy SM Lai ${ }^{1}$, FRCSEd (Ophth)

${ }^{1}$ Department of Ophthalmology, The University of Hong Kong, Hong Kong

${ }^{2}$ Department of Ophthalmology, Queen Mary Hospital, Hong Kong

${ }^{3}$ The University of Hong Kong

Correspondence and reprint requests:

Prof Jimmy Lai, Department of Ophthalmology, The University of Hong Kong, Room 301, Block B, Cyberport 4, 100 Cyberport Road, Hong Kong.

Email:laism@hku.hk

\section{Abstract}

Aim: To report the safety and efficacy of EX-PRESS glaucoma filtration device implantation in Chinese glaucoma patients.

Methods: Records of 38 eyes from 38 Chinese glaucoma patients who underwent EX-PRESS shunt implantation between August 2012 and June 2014 with at least 1 year of follow-up were retrospectively reviewed. Main outcome measures included intraocular pressure (IOP), visual acuity, success rate, number of topical antiglaucoma medications applied, and postoperative complications.

Results: The mean IOP reduced significantly from $25.8 \pm 12.4 \mathrm{mmHg}$ before surgery to $15.1 \pm 6.3 \mathrm{mmHg}$ at 1 year after surgery $(\mathrm{p}<0.005)$. Repeated measures ANOVA showed that IOP at all time points after surgery was significantly lower than IOP before surgery. $73.7 \%$ of patients achieved complete or qualified success.

Conclusions: EX-PRESS shunt implantation is safe and effective in Chinese patients.

Key words: Filtering surgery; Glaucoma

\section{Introduction}

The EX-PRESS glaucoma filtration device (Alcon Laboratories, Fort Worth [TX], USA) is a non-valved stainless steel magnetic resonance imaging-compatible device. ${ }^{1}$ With an external lumen of $400 \mu \mathrm{m}$ and internal lumen of $50 \mu \mathrm{m}$, it facilitates minimally invasive surgery and promotes the postoperative consistency and predictability of trabeculectomy. ${ }^{2-4}$ The EX-PRESS shunt was originally intended for subconjunctival implantation. Although this implant achieves significant reduction in intraocular pressure (IOP), complications related to excess outflow such as hypotony, flat anterior chamber, and suprachoroidal hemorrhage are frequent. ${ }^{5,6}$ Therefore, subconjunctival implantation has been replaced by implantation under a scleral flap. ${ }^{2}$

The latest P50 model of the EX-PRESS shunt has a vertical channel in the back plate intended to direct aqueous flow more posteriorly to create a more diffuse posterior bleb. Compared with trabeculectomy, EX-PRESS shunt implantation demonstrates comparable IOP-lowering efficacy, faster visual recovery, and fewer postoperative complications, ${ }^{7}$ as well as lower risk of postoperative hypotony. ${ }^{8}$ In addition, EX-PRESS shunt negates the need for iridectomy, resulting in less inflammation and shorter operation time. ${ }^{9}$ To the best of our knowledge, the surgical outcomes of EX-PRESS shunt implantation in the Chinese population have not been reported. Therefore, we evaluated the safety and efficacy of P50 EX-PRESS shunt implantation in Chinese patients.

\section{Methods}

The study was approved by the Institutional Review Board of The University of Hong Kong and was conducted in accordance with the Declaration of Helsinki. Medical records 
of Chinese glaucoma patients who underwent EX-PRESS shunt implantation between August 2012 and June 2014 at Queen Mary Hospital with at least 1 year of follow-up were retrospectively reviewed. For patients with bilateral EXPRESS shunt implantation, only information on the first eye was collected. Patients with cataract surgery within 1 year after EX-PRESS shunt implantation were excluded to avoid confounding IOP results. Patients with glaucoma surgery within 6 months before EX-PRESS shunt implantation were excluded to avoid the effect of scarring. Indications for EX-PRESS shunt implantation included suboptimal IOP control, disease progression despite maximally tolerated topical antiglaucoma medications, and topical medication intolerance.

Main outcome measures were IOP (measured by Goldman applanation), number of topical antiglaucoma medications applied, visual acuity, and postoperative complications requiring intervention. Each type of medication was counted as a single unit, with fixed combination eyedrops counted as two medications. Complete success was defined as IOP of $5-21 \mathrm{mmHg}$ in high-tension glaucoma or a $>20 \%$ decrease from preoperative IOP in normal-tension glaucoma, without antiglaucoma medications. Qualified success was defined using the same criteria, with patients on antiglaucoma medications achieving the target IOP. Failure was defined as hypotony with IOP of $<5 \mathrm{mmHg}$ or IOP of $>21 \mathrm{mmHg}$ despite topical medications, complete loss of light perception, or any further glaucoma drainage surgeries, excluding bleb needling.

All P50 EX-PRESS shunt implantations were performed by surgeons specializing in glaucoma surgery. Topical anesthesia with $0.4 \%$ oxybuprocaine was applied with or without subtenon anesthesia with $1 \%$ lidocaine. All conjunctival flaps were fornix based. Most shunts were implanted in the superonasal quadrant. In cases where the superonasal quadrant was not viable owing to conjunctival scarring or previous drainage surgery, the shunt was placed in other viable quadrants in the following order: superotemporal, inferotemporal, and inferior quadrant. A partial-thickness scleral flap of $4 \mathrm{~mm} \times 3 \mathrm{~mm}$ was created. Mitomycin C $(0.4$ $\mathrm{mg} / \mathrm{mL}$ ) was applied subconjunctivally and underneath the sclera flap for 2 minutes, followed by irrigation with $50 \mathrm{~mL}$ of balanced saline solution. A paracentesis wound was created with a $15^{\circ}$ stab knife at the temporal clear cornea. A 25 - or 27-gauge needle was used to enter the anterior chamber just anterior to the scleral spur, at the posterior aspect of the blue zone, creating a track parallel to the iris plane. The EX-PRESS shunt was implanted. The scleral flap was closed with two 10-0 nylon sutures, and the conjunctiva was closed with either 8-0 or 9-0 vicryl. Postoperative topical medications included $0.5 \%$ levofloxacin four times a day and $1 \%$ prednisolone acetate six to eight times a day. Patients were followed up at day 1 , week 1 , and months $1,3,6$, and 12 .

Normality of data was tested using Q-Q plots. Outcomes at 1 year after surgery were compared with preoperative values using the paired sample $t$-test for continuous variables and
Chi-squared test for categorical variables. Repeated measures ANOVA with Bonferroni post hoc test was used to compare continuous variables throughout the study period. Correlation between previous glaucoma surgery and the probability of success was determined using logistic regression analysis. Statistical significance was defined as $\mathrm{p}<0.05$. All statistical analyses were performed using SPSS (Windows version 20; IBM Corp, Armonk [NY], US).

\section{Results}

45 EX-PRESS shunt implantations were performed during the study period. Two of the patients were lost to follow-up. Five of the patients underwent bilateral implantations and data from the second surgery were excluded to avoid statistical overestimation secondary to inter-eye correlation. A total of 21 right eyes and 17 left eyes from 28 men and 10 women (mean age, 59 \pm 17 years) were included for analysis (Table 1). The most common diagnosis was primary open angle glaucoma $(44.7 \%)$, followed by uveitic glaucoma $(28.9 \%)$. Previous ocular surgery was common, with $57.9 \%$ having had cataract surgery and $23.7 \%$ having had failed filtration surgery.

The mean IOP decreased from $25.8 \pm 12.4 \mathrm{mmHg}$ before surgery to $15.1 \pm 6.3 \mathrm{mmHg}$ at 1 year after surgery $(\mathrm{p}<0.005$, paired $t$ test, Table 2). The lowest mean IOP $(9.2 \pm 6.0 \mathrm{mmHg})$ occurred approximately 1 week after surgery. The box and whisker plot revealed outliers that lay $>1.5$ times above the third quartile (Figure). Repeated measures ANOVA with and without outliers both showed that IOP at all time points after surgery was significantly lower than IOP before surgery

\begin{tabular}{|lc|}
\hline Table 1.Patient demographics & No. $(\%)$ \\
\hline Variable & $28(73.7)$ \\
\hline Sex & $10(26.3)$ \\
\hline Male & \\
\hline Female & $21(55.3)$ \\
\hline Eye & $17(44.7)$ \\
\hline Right & $17(44.7)$ \\
\hline Left & $11(28.9)$ \\
\hline Diagnosis & $3(7.9)$ \\
\hline Primary open angle glaucoma & $3(7.9)$ \\
\hline Uveitic glaucoma & $2(5.3)$ \\
\hline Primary angle closure glaucoma & $1(2.6)$ \\
\hline Congenital/childhood/juvenile glaucoma & $1(2.6)$ \\
\hline Normal-tension glaucoma & $7(18.4)$ \\
\hline Silicon oil-induced glaucoma & $4(10.5)$ \\
\hline Neovascular glaucoma & $22(57.9)$ \\
\hline Previous surgery & \\
\hline Cataract surgery & \\
\hline Trabeculectomies & \\
\hline Pars plana vitrectomies & \\
\hline Ahmed valves & \\
\hline
\end{tabular}


(Table 3). Patients who required a second glaucoma surgery within 1 year after of EX-PRESS shunt implantation were excluded from the ANOVA analysis.

The mean number of topical antiglaucoma medications applied was $3.3 \pm 1.5$ before surgery, $1.0 \pm 1.4$ at 6 months after

\begin{tabular}{|lc|}
\hline \multicolumn{2}{|l|}{ Table 2. Intraocular pressure $(\mathbf{I O P})$ at different time points } \\
\hline Time point & Mean \pm SD $(\mathbf{9 5 \%} \mathbf{C I}) \mathbf{I O P}, \mathbf{m m H g}$ \\
\hline Before surgery & $25.8 \pm 12.4(21.1-30.5)$ \\
\hline After surgery & \\
\hline 1 day & $10.1 \pm 6.4(7.7-12.6)$ \\
\hline 1 week & $9.2 \pm 6.0(7.0-11.5)$ \\
\hline 1 month & $10.6 \pm 4.6(8.8-12.3)$ \\
\hline 3 months & $12.3 \pm 4.6(10.5-14.0)$ \\
\hline 6 months & $13.7 \pm 4.8(12.0-15.6)$ \\
\hline 12 months & $15.1 \pm 6.3(12.7-17.5)$ \\
\hline
\end{tabular}

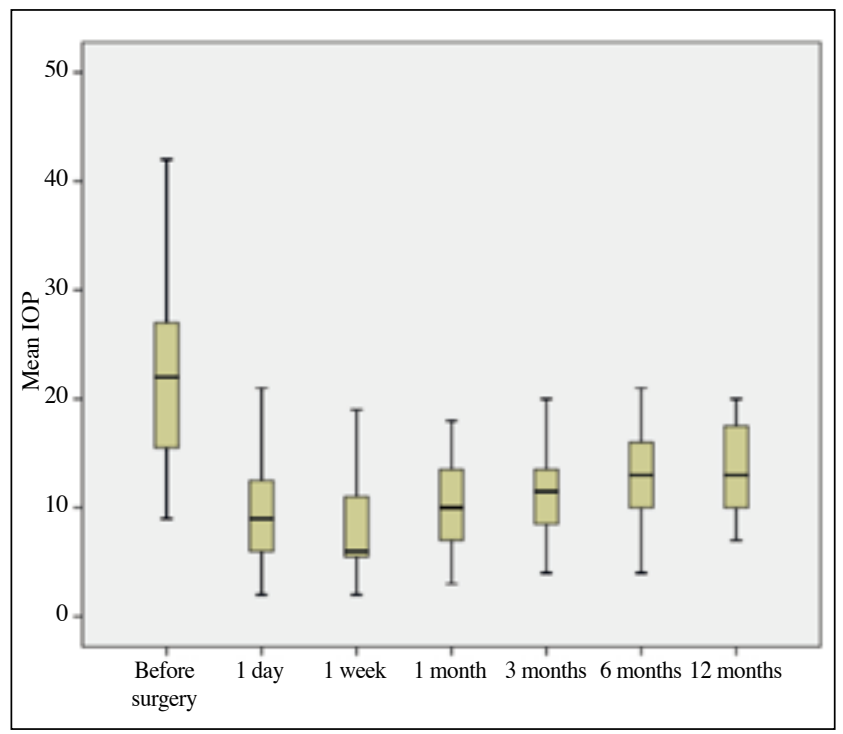

Figure. Box and whisker plot showing intraocular pressure (IOP) before surgery and at different time points after surgery surgery, and $1.2 \pm 1.3$ at 12 months after surgery $(\mathrm{p}<0.0005$, Wilcoxon signed rank test). $14(37 \%)$ patients required oral acetazolamide before surgery and none required it after surgery.

At 1 year after surgery, of 38 eyes, 14 (36.8\%) achieved a complete success, $14(36.8 \%)$ achieved a qualified success, and 10 failed. Of the 10 failed eyes, five had IOP of $>21$ $\mathrm{mmHg}$ despite medication and five underwent further glaucoma drainage surgeries (3 of which had secondary glaucoma). In subgroup analysis, of 17 patients with open angle glaucoma, $10(58.8 \%)$ achieved a complete success, five $(29.4 \%)$ achieved a qualified success, and two failed. Of 11 patients with uveitic glaucoma, four $(36.4 \%)$ achieved a complete success, three $(27.3 \%)$ achieved a qualified success, and four failed. Of three patients with primary angle closure glaucoma, two achieved a qualified success, and one failed. All four patients with neovascular or congenital glaucoma achieved a qualified success. The patient with silicon oil-induced glaucoma failed. The two patients with normal-tension glaucoma also failed. Logistic regression analysis showed no significant correlation between previous glaucoma surgery and the probability of success. No patients had hypotony or vision loss. Three patients required EXPRESS shunt removal (Table 4).

There were no significant differences before surgery and at 1 year after surgery in terms of visual acuity $(\log M A R$, $0.75 \pm 0.85$ vs $0.61 \pm 0.73, p=0.09$, paired $t$ test), cup-disc ratio $(0.67 \pm 0.2$ vs $0.69 \pm 0.2, p=0.27$, paired t test $)$, and mean deviation on Humphrey $24-2$ visual fields $(-15.3 \pm 9.0 \mathrm{~dB}$ vs $-18.4 \pm 9.7 \mathrm{~dB}, \mathrm{p}=0.45$, paired $t$ test).

\section{Discussion}

Trabeculectomy has been the standard surgical treatment for glaucoma since the 1970s. However, it is associated with a wide range of postoperative complications and a lower success rate in patients with secondary glaucoma, non-Caucasians, and young patients. ${ }^{10}$ Implantation of a tube shunt is an alternative option; however, shunts are associated with complications such as hypotony, diplopia, strabismus, tube erosion, and corneal decompensation. ${ }^{11}$ Compared with trabeculectomy, EX-PRESS shunt implantation achieve

\begin{tabular}{|c|c|c|c|c|}
\hline Repeated measures ANOVA & With outliers & P value & Without outliers & Pvalue \\
\hline Greenhouse-Geisser correction & $\mathrm{F}(2.282,63.9)=25.94$ & $<0.0005$ & $F(2.514,55.3)=23.6$ & $<0.0005$ \\
\hline Bonferroni post hoc test & Mean difference in IOP, $\mathrm{mmHg}$ & & $n$ difference in IOP, $\mathrm{m}$ & \\
\hline Before vs 1 day after surgery & 15.7 & $<0.0005$ & 13.0 & $<0.0005$ \\
\hline Before vs 1 week after surgery & 16.6 & $<0.0005$ & 14.5 & $<0.0005$ \\
\hline Before vs 1 month after surgery & 15.2 & $<0.0005$ & 12.0 & $<0.0005$ \\
\hline Before vs 3 months after surgery & 13.5 & $<0.0005$ & 11.0 & $<0.0005$ \\
\hline Before vs 6 months after surgery & 12.0 & $<0.0005$ & 9.2 & $<0.0005$ \\
\hline Before vs 12 months after surgery & 10.7 & $<0.0005$ & 8.3 & $<0.001$ \\
\hline
\end{tabular}




\begin{tabular}{|lc|}
\hline \multicolumn{2}{|l|}{ Table 4. Postoperative complications requiring interventions } \\
\hline Complication & No. $(\%)$ \\
\hline $\begin{array}{l}\text { Hypotony with shallow anterior chamber requiring } \\
\text { anterior chamber reformation with viscoelastic }\end{array}$ & $8(21.1)$ \\
\hline Bleb leak requiring conjunctival repair \\
\hline Antimetabolite injection \\
\hline \multicolumn{1}{|l|}{ Mitomycin C } \\
\hline 5 fluorouracil & $19(50)$ \\
\hline Needling with antimetabolite injection & $4(10.5)$ \\
\hline Inflammation requiring subtenon or oral steroids & $10(26.3)$ \\
\hline Suture lysis & $2(5.3)$ \\
\hline EX-PRESS shunt removal & $7(18.4)$ \\
\hline
\end{tabular}

similar efficacy, better tolerability, and fewer complications of hypotony and hyphema. ${ }^{12}$

Although the definition of success differs in different studies, the complete success rate of EX-PRESS shunt implantation at 1 year has been reported to be $60 \%$ to $87 \% .^{13-16}$ The success rate was reported to be $50 \%$ lower among non-Caucasians patients (mainly patients of African or Indian origin).$^{14}$ For trabeculectomy, outcomes have been reported to be similarly poor among Chinese ${ }^{17}$ and Malay ${ }^{18}$ patients. Other risk factors include young age, previous glaucoma surgery, and use of antiglaucoma medications. Glaucoma type was not found to be a risk factor for failure, but the sample comprised $>85 \%$ of open angle glaucoma cases and did not include secondary glaucoma or congenital glaucoma. ${ }^{14}$

In the present study, the complete success rate at 1 year was $36.8 \%$, which is lower than that reported in other studies. This may be attributed to differences in case mix. Open angle glaucoma only constituted $44.7 \%$ of our patients. The remainder included uveitic glaucoma (28.9\%), congenital or juvenile glaucoma (7.9\%), angle closure glaucoma (7.9\%), and other secondary glaucomas $(10.5 \%)$. These patients were at risk of trabeculectomy failure, and thus EX-PRESS shunt implantation assumed the role of augmented trabeculectomy and was an alternative to tube shunt implantation. The complete success rate of $58.8 \%$ among patients with open angle glaucoma is comparable with that reported in the literature.
Most of our patients had previous ocular surgery. However, logistic regression analysis showed no significant correlation between previous glaucoma surgery and the probability of success of the current surgery. This may be attributed to the small sample size. Of the three patients with angle closure glaucoma, two were pseudophakic prior to implantation (one of them required shunt exchange for malpositioned shunt) and one was phakic and achieved a qualified success. Although an open angle is usually a prerequisite for EX-PRESS shunt implantation, EX-PRESS shunts have been used in patients with angle closure glaucoma, usually in combination with phacoemulsification..$^{14,19}$ To the best of our knowledge, there are no studies on EX-PRESS shunt implantation in patients with phakic angle closure glaucoma. The success in patients with angle closure glaucoma suggests that EX-PRESS shunt implantation in the area free of synechiae closure may be a viable option. The incidence of hypotony has been reported to be lower after EX-PRESS shunt implantation than after trabeculectomy. ${ }^{8,19,20}$ One study reported a hypotony rate of $15.6 \%$ in the first week after EX-PRESS shunt implantation, but none of these eyes developed flat anterior chamber or lens corneal touch. ${ }^{19}$ This may be because the $50-\mu \mathrm{m}$ internal lumen diameter of the EX-PRESS shunt provides more resistance to aqueous outflow compared with the $750-\mu \mathrm{m}$ smallest sclera punch in trabeculectomy, thus providing better anterior chamber maintenance, even in cases of hypotony. ${ }^{3}$ None of our three patients with angle closure glaucoma developed hypotony, although two of them required anterior chamber reformation for shallow anterior chamber.

Limitations of our study include a heterogeneous sample, multiple surgeons, differences in anti-metabolite injection practices, and the retrospective nature.

\section{Conclusion}

EX-PRESS shunt implantation is effective and safe in primary open angle glaucoma and possibly in uveitic glaucoma. Further prospective and randomized controlled studies are recommended to clarify the efficacy of EX-PRESS shunt implantation in patients with angle closure glaucoma, neovascular glaucoma, or silicone oil-induced glaucoma.

\section{Declaration}

The authors have no conflicts of interest to disclose. This research received no specific grant from any funding agency in the public, commercial, or not-for-profit sectors.

\section{References}

1. Nyska A, Glovinsky Y, Belkin M, Epstein Y. Biocompatibility of the Ex-PRESS miniature glaucoma drainage implant. $J$ Glaucoma 2003;12:275-80. Crossref

2. Dahan E, Carmichael TR. Implantation of a miniature glaucoma device under a scleral flap. J Glaucoma 2005;14:98-102. Crossref

3. Sarkisian SR. The ex-press mini glaucoma shunt: technique and experience. Middle East Afr J Ophthalmol 2009;16:1347. Crossref

4. Rouse JM, Sarkisian SR Jr. Mini-drainage devices: the ExPRESS Mini-Glaucoma Device. Dev Ophthalmol 2012;50:905. Crossref

5. Wamsley S, Moster MR, Rai S, Alvim HS, Fontanarosa J. Results of the use of the Ex-PRESS miniature glaucoma 
implant in technically challenging, advanced glaucoma cases: a clinical pilot study. Am J Ophthalmol 2004;138:1049. 51. crossref

6. Traverso CE, De Feo F, Messas-Kaplan A, et al. Long term effect on IOP of a stainless steel glaucoma drainage implant (Ex-PRESS) in combined surgery with phacoemulsification. $\mathrm{Br}$ J Ophthalmol 2005;89:425-9. Crossref

7. Netland PA, Sarkisian SR Jr, Moster MR, et al. Randomized, prospective, comparative trial of EX-PRESS glaucoma filtration device versus trabeculectomy (XVT study). Am J Ophthalmol 2014;157:433-40.e3. Crossref

8. Marzette L, Herndon LW. A comparison of the Ex-PRESS mini glaucoma shunt with standard trabeculectomy in the surgical treatment of glaucoma. Ophthalmic Surg Lasers Imaging 2011;42:453-9. crossref

9. Freidl KB, Moster MR. ExPRESS shunt surgery: preferred glaucoma surgery in residency training? Surv Ophthalmol 2012;57:372-5. Crossref

10. Broadway DC, Chang LP. Trabeculectomy, risk factors for failure and the preoperative state of the conjunctiva. $J$ Glaucoma 2001;10:237-49. Crossref

11. Sarkisian SR Jr. Tube shunt complications and their prevention. Curr Opin Ophthalmol 2009;20:126-30. Crossref

12. Wang $W$, Zhou $M$, Huang $W$, Zhang X. Ex-PRESS implantation versus trabeculectomy in uncontrolled glaucoma: a metaanalysis. PLoS One 2013;8:e63591. Crossref

13. de Jong L, Lafuma A, Aguade AS, Berdeaux G. Five-year extension of a clinical trial comparing the EX-PRESS glaucoma filtration device and trabeculectomy in primary open-angle glaucoma. Clin Ophthalmol 2011;5:527-33. crossref

14. Mariotti C, Dahan E, Nicolai M, Levitz L, Bouee S. Longterm outcomes and risk factors for failure with the EX-press glaucoma drainage device. Eye (Lond) 2014;28:1-8. Crossref

15. de Jong LA. The Ex-PRESS glaucoma shunt versus trabeculectomy in open-angle glaucoma: a prospective randomized study. Adv Ther 2009;26:336-45. Crossref

16. Coupin A, Li Q, Riss I. Ex-PRESS miniature glaucoma implant inserted under a scleral flap in open-angle glaucoma surgery: a retrospective study [in French]. J Fr Ophtalmol 2007;30:1823. crossref

17. Tan C, Chew PT, Lum WL, Chee C. Trabeculectomy--success rates in a Singapore hospital. Singapore Med J 1996;37:5057.

18. Sharif FM, Selvarajah S. The outcome of trabeculectomy for primary glaucoma in adult patients in UKM. Med J Malaysia 1997;52:17-25.

19. Kanner EM, Netland PA, Sarkisian SR Jr, Du H. Ex-PRESS miniature glaucoma device implanted under a scleral flap alone or combined with phacoemulsification cataract surgery. J Glaucoma 2009;18:488-91. Crossref

20. Maris PJ Jr, Ishida K, Netland PA. Comparison of trabeculectomy with Ex-PRESS miniature glaucoma device implanted under scleral flap. J Glaucoma 2007;16:149. crossref 